\title{
PIPE LEAKAGEDETECTION USING HUMIDITY AND MICROPHONE SENSORS - A REVIEW
}

\author{
Ahmed Sachit Hashim ${ }^{1}$, Bogdan Grămescu², Constantin Niţu ${ }^{3}$ \\ 1,2,3 University POLITEHNICA of Bucharest \\ 313, Splaiul Independenţei, 060042-Bucharest, Romania \\ E-mail: ahmedhashim774@yahoo.com
}

\begin{abstract}
In view of the recent technological development in Europe in the field of wireless power transmission systems WPTs, one of the applications of these systems is the Radio Frequency identification RFIDs system. So, such a system can be used to send and receive the data, to and from sensors, to facilitate the task of detecting the leakage in pipelines, by using low-power wireless sensors, fixed like external devices and electronic devices on a mobile robot. Those sensors could be a humidity sensor for detecting oil leakage, and a microphone sensor to detect the gas leakage..
\end{abstract}

Keywords: Leak Detection, Humidity Sensor, Microphone Sensor, Mobile Robot.

\section{Introduction}

Aging and the continuous exposure to compressive stress and thermal stress in the pipelines during continuous operations, drive to the most common defects on pipes. The main reason for leakage in pipelines is the occurrence of corrosion at the first stage, the cracks occur as a second stage and then the leak, in the last stage. The reason is the poor maintenance by the oil and gas companies. The fact is that pipelines should be observed from time to time (period of maintenance) before the leak occur and cause a stop in production, preventing the risk occurrence. Therefore, for remote detection, early maintenance is used to prevent the leakage in the pipelines. The mobile robots could be a solution that can be used with various sensors. This paper presents the benefits of using a humidity sensor and a microphone sensor to provide real-time wireless capabilities and remote monitoring by mobile robots, for early detection of oil leakage or gas leakage in the external pipelines. The time and date of leakage occurrence can be determined by sudden occurrence of the gradual decrease in pressure within the pipes, using the latest communication network means and the use of appropriate sensors [1]. We can take advantage of the energy of Humidity Sensor and Microphone Sensor by using the active low-frequency range from the Radio Frequency identification RFIDs between $100 \mathrm{kHz}$ to $500 \mathrm{kHz}$ for its long characteristics and magnetic induction to effectively transmit and receive signals, to detect the presence of gas leak and oil leak from the coated external pipes [2]. During the development of technology in Europe, the solar transformers produced by the company Center, use transfer techniques ceramic CTTC. These transformers work to collect the solar energy to convert into DC to produce active frequencies for their radiation to be used in WPT. The WPT is one of the applications of RFIDs. So, the RFIDs can be used to send and receive the data, to and from sensors, to facilitate the task of detecting the leakage, by using low-power wireless sensors, fixed like external devices and electronic devices on a mobile robot. The sensors could be a humidity sensor for detecting oil leakage, and a microphone sensor to detect gas leakage on the external pipelines [3]. Experimentally, the location and size of the leakage in a gas pipeline using the microphone was detected with a significant increase in noise which is caused by the leakage. It was observed using the artificial neural network, depending on Radial Basis Function (RBF) Network (one of the artificial neural network application) [4]. The artificial signal and detection by using a microphone could be simulated, as a result of the pressure balance generated in the pipe by friction between the walls in the pipe. It is also the best way to detect leakage in gas pipelines [5]. The wireless system to detect the external leakage of gas and oil in the pipes using a humidity sensor and a microphone sensor was used in some applications. It is based on an Arduino microcontroller and it is a nondestructive technology (NDT). The data is obtained from the sensors, then transferred via ZigBee and then processed by a powerful software (for example LabVIEW). If it is necessary, the results can be transferred to a Web page [6]. Artificial neural network technology could be used, to detect gas leakage in pipelines using a microphone sensor to analyze the sounds of noise dynamics using different frequencies as input into the neural model. The size and location of the leakage from the model outputs 
were determined, and the results showed that method gives the detection accuracy $100 \%$ on the leakage [7]. Vibration waves that pass along the walls of pipes resulting from leaks was detected. After that, using FFT as a mathematical tool, it was calculated the dominant peak on the frequencies, where the frequencies range was about $20-200 \mathrm{~Hz}$ [8]. A wireless sensor network system WSN has been used to detect gas. The effectiveness of this system, its accuracy and speed in response to gas leakage, and the monitoring and assistance of the Environmental Meteorological Service have been demonstrated to detect the risks of gas diffusion in the atmosphere as well [9].

\section{Detect Leakage in Gas and Oil Pipelines}

Leakage is defined as a method of escape or the exit of the gas and the oil due to a-holes occurrence, due to exceeding the maximum permissible for overwork of pressure and temperature inside the pipelines. This type of behavior is undesirable, and it is a dangerous defect, causing significant losses in physical material (and economic losses). Leakage could also drive to enormous environmental disasters. Safe, continuous detection and adequate protection of pipes should be provided to prevent leakage to occur. Helped by the evolution of modern technology in the last two decades, the leakages in the pipes can be detected by using some remote sensors, to determine the location and size of the leakages. There are four important activities to reduce the occurrence of leakages in the gas and oil pipelines as follows: pressure management within the pipes; effective acceleration control; speed and quality repairs in the production; maintenance, repair, and replacement of damaged pipelines with regular periodic times.

\section{The Functional Description of Wireless Power Transmission Systems WPTs with Radio Frequency Identification System RFIDs}

Transmitter system is a device that generates a constant wave signal $\mathrm{CW}$ at $2.4 \mathrm{GHz}$, amplifies the RF signal, and then the amplified signal is emitted by an antenna.

Receiver system - the RF signal is received by an antenna, where the rectifier sys-tem converts the receiving signal $2.4 \mathrm{GHz}$ to a $\mathrm{DC}$ voltage signal. Of course it is necessary to have a battery in order to power the circuit with suitable voltages to enable it to accomplish the task.

\section{Benefits of Wireless Power Transmission Systems WPTs}

The advantages of wireless power transmission systems are based on the maximum RF efficiency to transmit, receive, amplify and convert DC signals as follows and as shown in figure.1.

- Increased energy transfer density.

- Generate and amplify the frequency signal and its radiation by an antenna.

- Generated and control the volume of energy required for the circuit.

- Reduce the range of harmonics.

- Specify the total size of antenna [10].

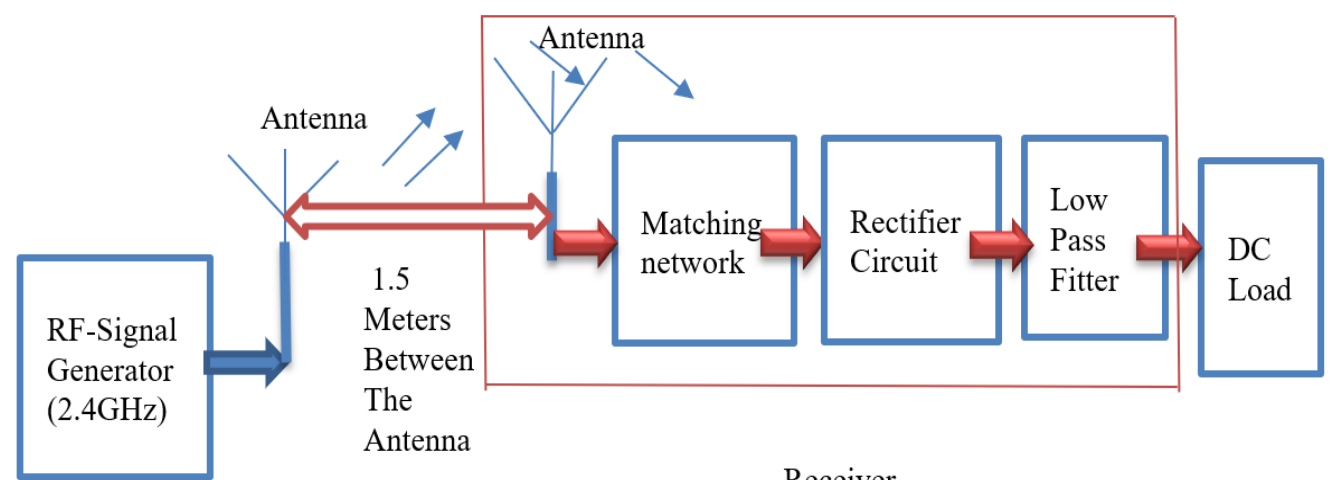

Transmitter

Receiver

Figure 1: The working WPTs with the RFIDs [10]

\section{Why the RFIDs System was Selected for Detection Purposes?}

RFIDs have been chosen because it has long characteristics and magnetic induction is good. The $\mathrm{RF}$ signal can be radiated effectively if the antenna dimensions are similar to the operating frequency wavelength. Where the frequencies are very low between $100 \mathrm{kHz}$ to $500 \mathrm{kHz}$, and because the wavelength of the operating frequency is in kilometers, therefore, the antenna must be well designed and installed with pre-calculated distances on the pipes insulators. 
For example, in North America, antenna transmission capacity was $36 \mathrm{dBm}$, where most RFID tags require microchips of $10 \mathrm{dBm}$ or better for the purpose of obtaining good and accurate signal response. So, the low frequency system RFID could operate with a humidity sensor and microphone sensor, and it must match the high-precision ideal resistance between the RFID chip and tag used in the humidity sensor, for example. The signal from the RFID system is proportional to the relative humidity resulting from detecting on crude oil leakage on the external pipes.

\section{DHT22 Humidity Sensor}

Moisture is an unwanted contaminant and is capable of penetrating virtually any surface, including metals such as copper, aluminum, bronze, and carbon steel.

Therefore, it is important first to accurately measure moisture content, to control or remove unwanted moisture. By understanding the time and how to measure and manage humidity, we are able to improve product quality, reduce equipment damage, save energy, reduce costs, and meet the obligations to provide protection for pipes in particular. The negative side of moisture includes condensation, corrosion, cracking, leakage, pollution and many hazards. Obviously, moisture has the potential to cause expensive problems.

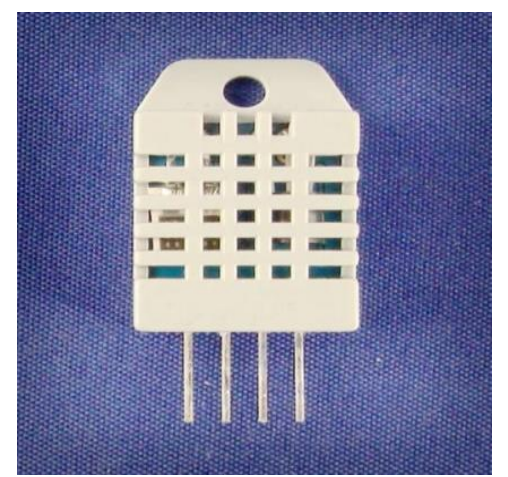

Figure 2: DHT22 Humidity Sensor [6].

Description of the DHT22 moisture sensor digital signal with calibration output. It uses an exclusive technology for digital signal collection and moisture sensor technology, ensuring its reliability and stability. Its sensor elements are connected to an 8-bit mono chip; they are installed on a computer or on mobile robots. Small size, low power consumption and long-distance transmission (20 m) made DHT22 suitable and easy to use.

DHT22 Moisture Sensor Operation Specifications:

- Energy processed

The voltage must be $3.3-6 \mathrm{~V}$ DC. When power is supplied to the sensor, do not send any instructions to the sensor within one second to pass the unstable situation. One $100 \mathrm{nF}$ capacitor can be added between VDD and GND to filter the waves, as shown below in the table.1.

Table 1. The technical specification for the DHT22 moisture sensor [6].

\begin{tabular}{|l|l|}
\hline Power supply & $3.3-6 \mathrm{~V} \mathrm{DC}$ \\
\hline Output signal & digital signal via single-bus \\
\hline Sensing element & Polymer capacitor \\
\hline Operating range & humidity $0-100 \% \mathrm{RH}$; temperature $-40 \sim 80^{\circ} \mathrm{C}$ \\
\hline Accuracy & humidity $+-2 \% \mathrm{RH}(\mathrm{Max}+-5 \% \mathrm{RH})$; temperature $<+-0.5{ }^{\circ} \mathrm{C}$ \\
\hline Resolution or sensitivity & humidity $0.1 \% \mathrm{RH}$; temperature $0.1{ }^{\circ} \mathrm{C}$ \\
\hline Repeatability & humidity $+-1 \% \mathrm{RH}$; temperature $+-0.2^{\circ} \mathrm{C}$ \\
\hline Humidity hysteresis & $+-0.3 \% \mathrm{RH}$ \\
\hline Long-term Stability & $+-0.5 \% \mathrm{RH} / \mathrm{year}$ \\
\hline Sensing period & Average: $2 \mathrm{~s}$ \\
\hline Dimensions & small $14 \mathrm{~mm} \times 18 \mathrm{~mm} \times 5.5 \mathrm{~mm} ;$ big $22 \mathrm{~mm} \times 28 \mathrm{~mm} \times 5 \mathrm{~mm} ;$ \\
\hline
\end{tabular}

- $\quad$ The system of Communications

Data is used to communicate between MCU and DHT22, costing $5 \mathrm{mS}$ for one-time communication. The data consists of the integral and decimal part. That is, the DHT22 sensor sends the data slightly higher or lower, that is, DATA = Integrated RH data 8 bits +8 Decimal RH data +8 bits Integrated $\mathrm{T}$ data + 8 bits $\mathrm{T}+8$ bits Optional value If data transfer is appropriate, the total checksum is the last 8 bits of 8 data integrated $\mathrm{RH}+8$ bits decimal data decimal $\mathrm{RH}$ +8 bits integrated $\mathrm{T}$ data +8 decimal bits. When the MCU sends the start signal, the DHT22 sensor changes from the low power consumption mode to the power-on mode. When the MCU finishes sending the start signal, the DHT22 sensor sends a response signal from 40-bit data that reflects the occurrence of relative humidity, as shown below in the table. 2 . 
Table 2.The Electrical Characteristics of the DHT22 moisture sensor [6].

\begin{tabular}{|c|c|c|c|c|c|}
\hline Item & Condition & Min. & Typical & Max. & Unit \\
\hline Power supply & DC & 3.3 & 5 & 6 & $\mathrm{~V}$ \\
\hline $\begin{array}{c}\text { Current } \\
\text { supply }\end{array}$ & Measuring & 1 & & 1.5 & $\mathrm{~mA}$ \\
\cline { 2 - 6 } & Stand-by & 40 & Null & 50 & $\mathrm{uA}$ \\
\hline $\begin{array}{c}\text { Collecting } \\
\text { period }\end{array}$ & Second & & 2 & & $\begin{array}{c}\text { Secon } \\
\mathrm{d}\end{array}$ \\
\hline
\end{tabular}

\section{- Working conditions}

The DHT22 sensor works in a good and stable condition when the temperature is between $50{ }^{\circ} \mathrm{C}$ and $60{ }^{\circ} \mathrm{C}$, humidity $<10 \% \mathrm{RH}$ for two hours. The DHT22 sensor works in a good and stable condition when the temperature is between 20 and $30{ }^{\circ} \mathrm{C}$, humidity $>70 \%$ RH for 5 hours. Relative humidity is strongly dependent on temperature, which is why we use temperature compensation technology to ensure RH measurement accuracy.

Therefore, we should focus on the work of the sensor DHT22 humidity with the recommended temperature and humidity as above, as shown below in figure.3.

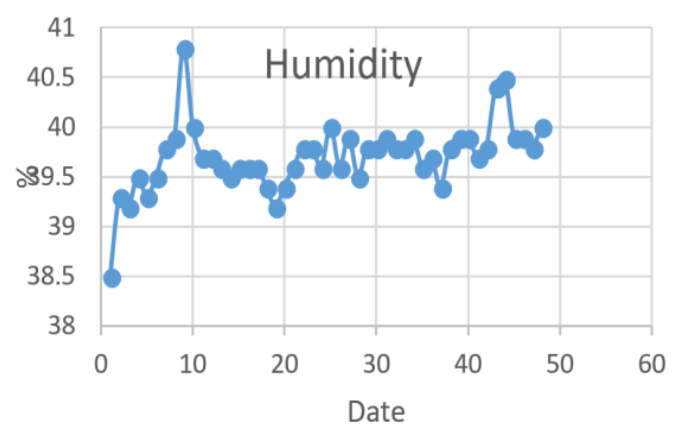

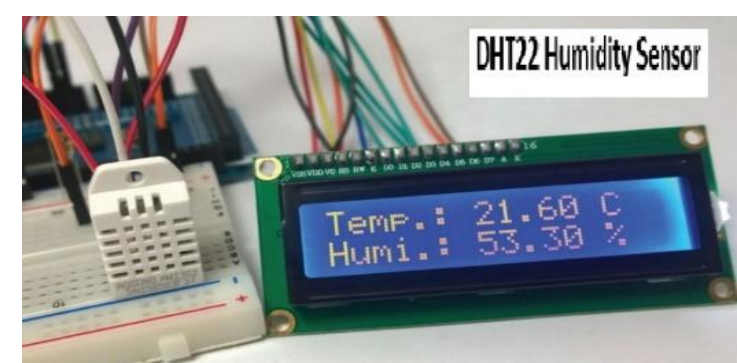

Figure 3: The reading of moisture and temperature

The following graph of the DHT22 Humidity Sensor reads the relative humidity and the temperature $\left({ }^{\circ} \mathrm{C}\right)$, as can be observed in Figure.4,a, and Figure.4,b, respectively.

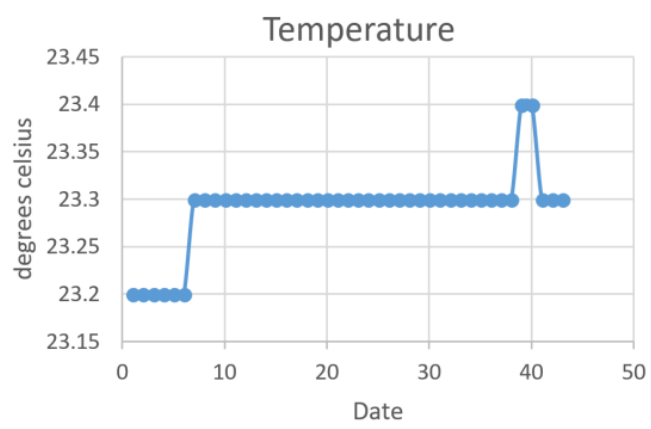

Figure 4: DHT22 Humidity Sensor reads the humidity (left); b. the temperature (right)

\section{MQ-6 Gas Microphone Sensor}

The microphone sensor detects and monitors the leakage resulting from gas mass flow at high speed and high pressure, because of the high sound and pipe vibration occurrence due to the leakage. Pipeline leakage, lead to a reduction the pressure inside the pipes, loss, and degradation of products, environmental pollution, and many risks.

The technical data for MQ-6 gas microphone Sensor is as follows:

- the features

It has high sensitivity to LPG, ISO, butane, and propane. Small sensitivity to alcohol and smoke. Fast response. A stable and long life; Circuit design in a good mode with a simple Specifications.

- operation and control detection sensitivity with the MQ-6 gas microphone sensor

The value of MQ- 6 resistance is affected by different types of gases and their different concentrations.
Therefore, when using this device, the sensitivity must be adjusted and calibrated when it detects $1000 \mathrm{ppm}$ of the LPG concentration in its environment. The load resistance value (RL) used is about $20 \mathrm{~K}(10 \mathrm{~K}$ to $47 \mathrm{~K})$. When measuring accurately, the appropriate warning point for the gas detector should be determined by considering the effect of temperature and humidity as shown below in the figure.5.

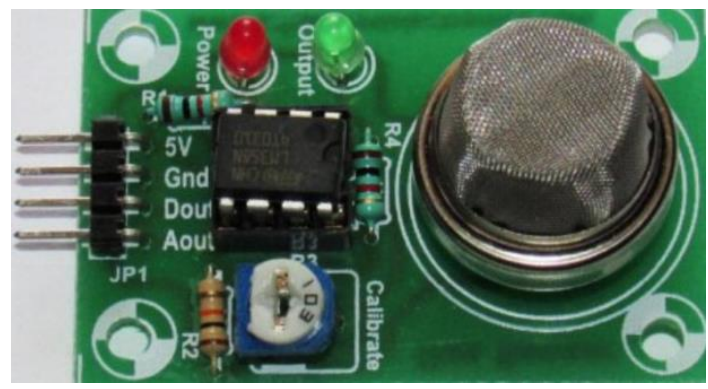

Figure 5: MQ-6 microphone sensor for gas. 


\section{Mobile Robot for Leakage Detection}

For the leakage detection it is necessary to have a mobile support for sensors. Next rows present the external pipe locomotion of a robot for inspection and monitoring of the pipelines for use in oil and gas industry (Figure 6). During regular motion, the robot resembles to a crank-slider mechanism with equal lengths of the crank and rod, while for stepping over flanges it becomes an open chain with double actuated leverage. It could be used for inspection and monitoring purposes, detecting the most common defects on the horizontal and vertical external pipes.

It needs to be able for receiving and transmitting data to and from the worker.

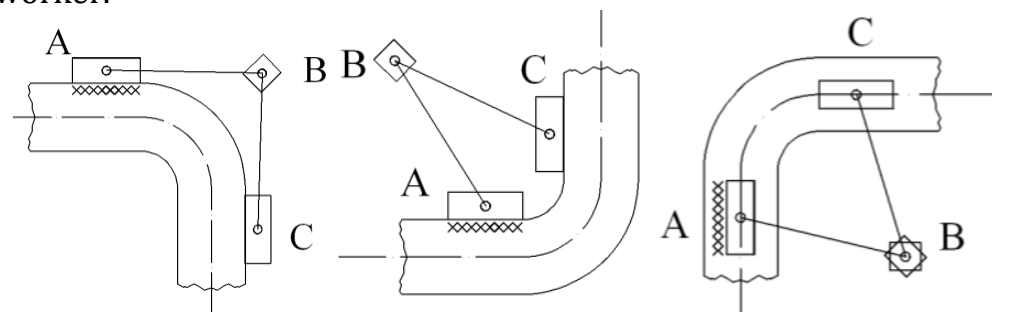

Figure 7: Robot at turns down and up; Robot at turns right and left

\section{Conclusion}

WPTs using radio frequency identification (RFID) systems are efficient, reliable and provide highresolution. Using such a system, a mobile robot could have succes in detecting the most common defects above the external pipes. The robot should have the ability to carry and install the external devices and electronic devices on his body. The humidity sensor has been used to detect the crude oil leakage and the microphone sensor to detect the leakage of gas on the external pipelines.

\section{References}

[1] Ejofodomi.O, Ofualagba.G,"Exploring the Feasibility of Robotic Pipeline Surveillance for Detecting Crude Oil Spills in the Niger Delta", New Brunswick, Canada, IJUSEng, Vol. 5, No. 3, 38-52; 2017.

[2] Nasir. A, Soong. B.H, Qaraqe .K.A,"RFID in-pipe moisture sensing system for Oil and Gas quality monitoring in Qatar", Texas A\&M University at Qatar and Nanyang Technological University; 2013.

[3] Carvalho .N.B, Senior Member, IEEE, Georgiadis .A, Senior Member, IEEE, et al," Wire-less Power Transmission: R\&D Activities within Europe", VOL. 62, NO. 4; 2014.

[4] Santos.R.B, Ruppb.M, Santiago J. Bonzi.S.J, Filetia.A.M.F, "Comparison Between Multi-layer Feedforward Neural Networks and a Radial Basis Function Network to Detect and Locate Leaks in
Mobile robot has the ability to move along the pipes, to negotiate the curves of the pipes. (Figure 7)

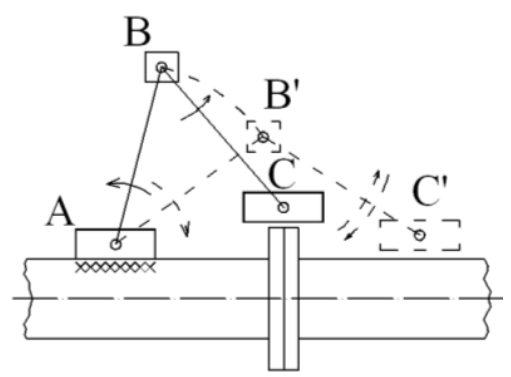

Figure 6: Mobile robot locomotion and jumping on the obstacle to detection.
Pipelines Transporting Gas", the Technical University of Berlin in Germany and the University of Campinas in Brazil, CHEMICAL ENGINEERING-TRANSACTIONS, VOL. 32; 2013.

[5] Adnan.N F, Ghazali.M F, Amin.M M, and Hamat. A. M. A,"Leak detection in gas pipeline by acoustic and signal processing - A review", IOP Publishing, (ICMER), Malaysia; 2015.

[6] Adsul.S, Sharma.A.K, Mevekari.R.G,"Development of Leakage Detection System", Inter-national Conference on Automatic Control and Dynamic Optimization Techniques (ICACDOT), International Institute of Information Technology ( ${ }^{2}$ IT), Pune, India; 2016.

[7] Santos.R. B, de Sousa.E. O, da Silva.F. V, da Cruz.S. L and A. M. F. Fileti.A. M. F,"Real-time Monitoring Of Gas Pipeline Through Artificial Neural Networks", School of Chemical Engineering, University of Campinas, Brazil; 2013.

[8] Karkulali.P, Himanshu. M,"Leak Detection in Gas Distribution Pipelines using Acoustic Impact Monitoring", Energy Research Institute and School of Electrical \& Electronics En-gineering in Nanyang Technological University, Singapore.

[9] Luo.Q, XiaoranGuo.X, Wang.Y, XufengWei.X,"Design of Wireless Monitoring System for Gas Emergency Repairing", University of Beihang, Beijing, China, IEEE; 2016.

[10] Dr.pro. Shastry.P and members are Baliss.E, Sanchez.S, \&Hoge.T, "Wireless Power Transfer System WPTs, Department of Electrical and Computer Engineering, Bradley University in Peoria (Illinois), USA; 2013. 